\title{
SLC1A3 (EAAT1) is over-expressed in brain metastatic breast cancer.
}

Shahan Mamoor

East Islip, NY USA ${ }^{1}$

1shahanmamoor@gmail.com

Brain metastases are a clinical problem in patients with breast cancer ${ }^{1-3}$. We mined published microarray data $^{4,5}$ to discover genes associated with brain metastasis in patients with brain metastatic breast cancer.

We found that the gene encoding the glutamate transport SLC1A3 was among the genes most differentially expressed in the brain metastases of patients with brain metastatic breast cancer. SLC1A3, and its function in the transport of glutamate 6 may be relevant to the biology underlying metastasis of competent breast cancer clones to the brain.

Keywords: breast cancer, brain metastases, central nervous system metastases, SLC1A3, systems biology of breast cancer, targeted therapeutics in breast cancer. 
One report described a 34\% incidence of central nervous system metastases in trastuzumabtreated patients with breast cancer ${ }^{2}$. This alarmingly high frequency of CNS metastasis events demands an enhanced understanding of the transcriptional makeup of brain metastatic tissues to support identification of therapeutic targets. We performed a global comparative analysis of transcription in the brain metastases of patients with brain metastatic breast cancer ${ }^{4,5}$. We discovered significant differential and over-expression of SLC1A3 in brain metastases from patients with brain metastatic breast cancer.

\section{Methods}

We used datasets GSE1259894 and GSE526045 for this global differential gene expression analysis of brain metastatic breast cancer in conjunction with GEO2R. GSE125989 was generated using Affymetrix Human Genome U133A 2.0 Array technology with $n=16$ primary tumors from patients with breast cancer and $n=16$ brain metastases from patients with brain metastatic breast cancer. GSE52604 was generated using Whole Human Genome Microarray 4x44K G4112F technology with $n=10$ normal breast tissues and $n=35$ brain metastases from patients with breast cancer, analysis performed using platform GPL6480. The Benjamini and Hochberg method of $p$-value adjustment was used for ranking of differential expression but raw $p$-values were used to assess statistical significance of global differential expression. Log-transformation of data was auto-detected, and the NCBI generated category of platform annotation was used. A statistical test was performed to evaluate whether SLC1A3 gene expression was significantly between primary breast tumors and brain metastases in humans with breast cancer using a two-tailed, unpaired t-test with Welch's correction. We used PRISM for all statistical analyses of differential gene expression in human breast cancer (Version 8.4.0)(455).

\section{Results}

We performed global comparative transcriptome analysis of the primary and metastatic tumor tissues of patients with brain metastatic breast cancer using published microarray data 4,5 to describe the transcriptional landscape of brain metastasis in human breast cancer in an unbiased fashion and for the discovery of novel therapeutic targets.

\section{SLC1A3 is differentially expressed in the brain metastases of patients with brain metastatic breast cancer.}

We identified the gene encoding SLC1A3 as among the genes whose expression was most significantly different in the brain metastases of patients with brain metastatic breast cancer ${ }^{4}$ (Table 1). When sorting each of the genes expressed in brain metastases based on significance of difference as compared to the primary tumors of patients with brain metastatic breast cancer, SLC1A3 ranked 77 out of 22297 total transcripts (Table 1), equating to $99.7 \%$ differential expression. Differential expression of SLC1A3 in the brain metastases of patients with brain metastatic breast cancer was statistically significant (Table $1 ; p=1.96 \mathrm{E}-04)$.

Cross-validation of differentially expressed genes across datasets in cancer can be difficult and more challenging than when cross-validating across organs and sorted cell populations due to higher levels of heterogeneity in tumors, and differing methods of tumor sampling between laboratories. Thus, we asked whether we could validate differential expression of SLC1A3 when comparing brain metastases from patients with breast cancer to normal breast tissue as opposed to primary tumors of the breast. Comparison of global gene expression profiles of 10 normal breast tissues to 35 brain metastases ${ }^{5}$ revealed that SLC1A3 was among the genes whose expression was most significantly different transcriptome-wide between the breast and brain metastases in patients with breast cancer (Table 2). 
When sorting each of the genes expressed in brain metastases based on significance of difference in expression between brain metastases and normal breast tissues, SLC1A3 ranked 460 out of 41093 total transcripts (Table 2), equating to $98.9 \%$ differential expression. Differential expression of SLC1A3 in brain metastases from patients with brain metastatic breast cancer in this dataset was statistically significant (Table $2 ; p=6.67 \mathrm{E}-09$ ).

\section{SLC1A3 is expressed at significantly higher levels in the brain metastases of patients with brain metastatic breast cancer.}

We obtained exact mRNA expression levels for SLC1A3, in primary tumors of the breast and in brain metastasis of patients with brain metastatic breast cancer to determine magnitude and direction of change in SLC1 A3 expression in brain metastatic tissues. SLC1A3 was expressed at higher levels in the brain metastases of patients with breast cancer as compared to primary tumors of the breast, and this difference was statistically significant (Figure $1 ; p=0.0013$ ). We calculated a mean fold change of $1.30 \pm$ 0.25 in SLC1A3 expression when comparing primary tumors of the breast to brain metastatic tissues in patients with breast cancer (Table 1).

Thus, by mining published microarray data 4,5 in an unbiased and systematic fashion, we identified SLC1A3 as among the genes whose expression was most different in the brain metastases of patients with breast cancer both when compared to primary tumors of the breast and to the normal breast; SLC1A3 was expressed at significantly higher levels in brain metastases as compared to primary tumors of the breast.

\section{Discussion}

SLC1A3 is a Na+-dependent glutamate transporter expressed in glial cells ${ }^{6}$. Also known as EAAT $1^{7}$ and GLAST ${ }^{8}$, SLC1A3 is expressed in tissues throughout the nervous system including the cerebellum $^{7}$, ventricular zone ${ }^{8}$, retina ${ }^{9}$, and cochlea ${ }^{10}$. SLC1A3 has also been described to function in uptake of aspartate ${ }^{7}$. In the hair follicle and tissues associated with hair, cells of the interfollicular epidermis and sebaceous gland express SLC1A3, and expression of SLC1A3 is up-regulated in the bulge and outer root sheath during growth of hair; SLC1A3 was described as a marker of progenitor cells of the skin associated with the hair ${ }^{11}$. In the context of cancer, MDA-MB-468 breast cancer cells express relatively high levels of SLC1A3 protein, and deletion of SLC1A3 in the MDA-MB-468 cell line resulted in significantly decreased tumor volume when injected subcutaneously into BALB/c nude mice ${ }^{12}$. p53 could induce the expression of SLC1A3, supporting ability of cells to proliferate under conditions of glutamine insufficiency ${ }^{12}$. SLC1A3 may also contribute to the electron transport chain, as depletion of SLC1A3 in HCT116 cells reduced oxygen consumption rate under conditions of glutamine starvation ${ }^{12}$. Aspartate can be utilized to generate glutamine under conditions of glutamine starvation through the malate-aspartate shuttle involving the cytosolic and mitochondrial transaminases GOT1 and GOT2; as such, depletion of GOT1 or GOT2 impaired the ability of cells expressing wild-type p53 to proliferate under conditions of glutamine starvation but not in glutamine sufficiency ${ }^{12}$. SLC1A3 was found to be expressed at higher levels in gastric cancer as compared to normal tissues, and high expression of SLC1A3 was correlated with decreased overall patient survival ${ }^{13}$. In gastric cancer cell lines MKN45 and NCI-N87, expression of SLC1A3 supported production of ATP and lactate, and promoted phosphorylation of $\mathrm{AKT}^{13}$. Tumors of Balb/c nude mice xenografted with AGS gastric cancer cells were significantly decreased in size when SLC1A3 was depleted ${ }^{13}$. Thus, SLC1A3 can function in transport of glutamate in normal cells and in cancer, and SLC1A3 has been described to possess the ability to support cell proliferation.

We report here that SLC1A3, also known as EAAT1/GLAST, is among the genes whose expression is most different in the brain metastases of patients with brain metastatic brain cancer when compared to primary tumors of the breast. SLC1A3, through its ability to support transport of glutamate and to contribute to cellular metabolism, may be important for metastasis of breast cancer cells to the brain. 


\section{References}

1. Lin, N.U., Amiri-Kordestani, L., Palmieri, D., Liewehr, D.J. and Steeg, P.S., 2013. CNS metastases in breast cancer: old challenge, new frontiers.

2. Bendell, J.C., Domchek, S.M., Burstein, H.J., Harris, L., Younger, J., Kuter, I., Bunnell, C., Rue, M., Gelman, R. and Winer, E., 2003. Central nervous system metastases in women who receive trastuzumab-based therapy for metastatic breast carcinoma. Cancer, 97(12), pp.2972-2977.

3. Tsukada, Y., Fouad, A., Pickren, J.W. and Lane, W.W., 1983. Central nervous system metastasis from breast carcinoma autopsy study. Cancer, 52(12), pp.2349-2354.

4. Iwamoto, T., Niikura, N., Ogiya, R., Yasojima, H., Watanabe, K.I., Kanbayashi, C., Tsuneizumi, M., Matsui, A., Fujisawa, T., Iwasa, T. and Shien, T., 2019. Distinct gene expression profiles between primary breast cancers and brain metastases from pair-matched samples. Scientific reports, $9(1)$, pp. $1-8$.

5. Salhia, B., Kiefer, J., Ross, J.T., Metapally, R., Martinez, R.A., Johnson, K.N., DiPerna, D.M., Paquette, K.M., Jung, S., Nasser, S. and Wallstrom, G., 2014. Integrated genomic and epigenomic analysis of breast cancer brain metastasis. PloS one, 9(1), p.e85448.

6. Hagiwara, T., Tanaka, K., Takai, S., Maeno-Hikichi, Y., Mukainaka, Y. and Wada, K., 1996. Genomic organization, promoter analysis, and chromosomal localization of the gene for the mouse glial highaffinity glutamate transporter Slc1a3. Genomics, 33(3), pp.508-515.

7. Arriza, J.L., Fairman, W.A., Wadiche, J.I., Murdoch, G.H., Kavanaugh, M.P. and Amara, S.G., 1994. Functional comparisons of three glutamate transporter subtypes cloned from human motor cortex. Journal of Neuroscience, 14(9), pp.5559-5569.

8. Shibata, T., Yamada, K., Watanabe, M., Ikenaka, K., Wada, K., Tanaka, K. and Inoue, Y., 1997. Glutamate transporter GLAST is expressed in the radial glia-astrocyte lineage of developing mouse spinal cord. Journal of Neuroscience, 17(23), pp.9212-9219.

9. Diaz, C.M., Macnab, L.T., Williams, S.M., Sullivan, R.K. and Pow, D.V., 2007. EAAT1 and D-serine expression are early features of human retinal development. Experimental eye research, 84(5), pp. 876-885.

10.Hakuba, N., Koga, K., Gyo, K., Usami, S.I. and Tanaka, K., 2000. Exacerbation of noise-induced hearing loss in mice lacking the glutamate transporter GLAST. Journal of Neuroscience, 20(23), pp. 8750-8753.

PAGE 4 
11.Reichenbach, B., Classon, J., Aida, T., Tanaka, K., Genander, M. and Göritz, C., 2018. Glutamate transporter Slc1a3 mediates inter-niche stem cell activation during skin growth. The EMBO journal, 37(9), p.e98280.

12.Tajan, M., Hock, A.K., Blagih, J., Robertson, N.A., Labuschagne, C.F., Kruiswijk, F., Humpton, T.J., Adams, P.D. and Vousden, K.H., 2018. A role for p53 in the adaptation to glutamine starvation through the expression of SLC1A3. Cell metabolism, 28(5), pp.721-736.

13.Xu, L., Chen, J., Jia, L., Chen, X., Awaleh Moumin, F. and Cai, J., 2020. SLC1A3 promotes gastric cancer progression via the PI3K/AKT signalling pathway. Journal of Cellular and Molecular Medicine.

PAGE 5 


\begin{tabular}{r|l|l|l|l|l|l|l} 
Rank & ID & p-value & t & B & FC & Gene & Gene name \\
\hline 77 & 202800_at & $1.96 E-04$ & 4.1669206 & 0.6991 & $1.30 \pm 0.25$ & SLC1A3 & $\begin{array}{l}\text { solute carrier family } \\
1 \text { member 3 }\end{array}$ \\
\hline
\end{tabular}

Table 1: SLC1A3 is differentially expressed in brain metastases in brain metastatic breast cancer.

Rank of differential expression, probe ID, $p$-value with respect to differential expression, $t$, a moderated tstatistic, B, the log-odds of differential expression between the two groups compared, fold change (FC) of SLC1A3 expression between the brain metastases of patients with breast cancer and primary tumors of the breast, gene and gene name are listed in this chart. 
Figure 1: SLC1A3 is expressed at significantly higher levels in the brain metastases of patients with brain metastatic breast cancer.

The mRNA expression level of SLC1A3 in primary tumors of the breast (left) and in the brain metastases of brain metastatic breast cancer patients (right) is graphically represented here with mean SLC1A3 levels marked and the result of a statistical test evaluating difference in mRNA expression level between brain metastases and primary tumors of the breast, a $p$-value, listed above. 